\title{
The prevalence effect in two-dimensional stimulus-response compatibility is a function of the relative salience of the dimensions
}

\author{
KIM-PHUONG L. VU and ROBERT W. PROCTOR \\ Purdue University, West Lafayette, Indiana
}

\begin{abstract}
When stimulus-response (S-R) sets vary along horizontal and vertical dimensions, a right-left prevalence effect is often obtained in which the horizontal compatibility effect is larger than the vertical compatibility effect. $\mathrm{Vu}$ and Proctor (2001) showed that the prevalence effect varies as a function of the dimension made salient by the response configuration. A salient features coding interpretation of this result implies that manipulating the salience of the stimulus display should produce similar results and that S-R translation should be fastest when salient features of the stimulus and the response sets correspond. Experiment 1 manipulated spatial proximity to make the vertical or the horizontal stimulus dimension salient. Neutral displays yielded a typical right-left prevalence effect, and this effect was enhanced by horizontal-salient displays and eliminated by vertical-salient displays. Experiments 2 and 3 showed that the benefit for horizontal (or vertical) compatibility was larger when the salient features of both the stimulus and the response sets emphasized the horizontal (or the vertical) dimension than when only one did. The results support salient features coding as an explanation for the prevalence effect obtained with two-dimensional S-R arrangements.
\end{abstract}

Stimulus-response compatibility (SRC) refers to the fact that people perform better with certain mappings of stimuli to responses than with others (see, e.g., Hommel \& Prinz, 1997; Proctor \& Reeve, 1990). Most studies have used stimulus-response (S-R) sets that varied along a single dimension, such as two-choice tasks involving left and right spatial locations (e.g., Shaffer, 1965). In these tasks, reaction times (RTs) are faster when the left response is made to the left stimulus and the right response to the right stimulus than when the S-R mapping is reversed (e.g., Proctor \& Dutta, 1993). This relation is also observed when the stimuli and the responses are arrayed along the vertical dimension, with better performance when the top response is made to the top stimulus and the bottom response to the bottom stimulus than when the S-R mapping is reversed (Nicoletti \& Umiltà, 1984; Vu, Proctor, \& Pick, 2000).

When studied in isolation, horizontal and vertical SRC effects are of similar magnitude (Vu et al., 2000). However, for situations in which S-R sets vary along both the horizontal and the vertical dimensions simultaneously, the SRC effect obtained for one dimension can be larger than that obtained for the alternative dimension (Vu \& Proctor, 2001). The form of dominance that is usually obtained is

We thank Romeo Chua, Yvonne Lippa, and an anonymous reviewer for thoughtful comments on a previous version of the manuscript. This research also benefited from valuable suggestions made by Jim Nairne, David Pick, and Howard Zelaznik. Correspondence concerning this article should be sent to K.-P. L. Vu, Department of Psychological Sciences, Purdue University, West Lafayette, IN 47907-1364 (e-mail: kvu@psych.purdue.edu). that of right-left prevalence, in which the horizontal dimension dominates the vertical one (Nicoletti \& Umiltà, 1984, 1985; Nicoletti, Umiltà, Tressoldi, \& Marzi, 1988). However, top-bottom prevalence is obtained for situations in which response configurations make the vertical dimension more salient (Vu \& Proctor, 2001).

\section{Two-Dimensional Compatibility}

Nicoletti and Umiltà (1984, 1985; Nicoletti et al., 1988) evaluated two-dimensional SRC effects by presenting stimuli along a diagonal in the top-left/bottom-right or top-right/bottom-left corners of a display panel paired with four similarly arranged response locations. By varying the stimulus locations and response positions, along with the S-R mapping, it was possible to have conditions that were compatible on one, both, or neither of the two dimensions (see Figure 1). For the condition with compatibility on both dimensions (both condition), the top-left stimulus was mapped to the top-left response and the bottomright stimulus to the bottom-right response; the opposite S-R mapping was used for the condition with compatibility on neither dimension (neither condition). Similarly, for the condition with vertical compatibility alone (vertical condition), the top-left stimulus was mapped to the top-right response and the bottom-right stimulus to the bottom-left response; the opposite mapping was used for the condition with horizontal compatibility alone (horizontal condition).

Nicoletti and Umiltà $(1984,1985)$ found that that the conditions with horizontal and vertical compatibility alone yielded a benefit, relative to the neither condition. On the basis of this finding, they suggested that codes are formed along more than one dimension at stimulus presentation 


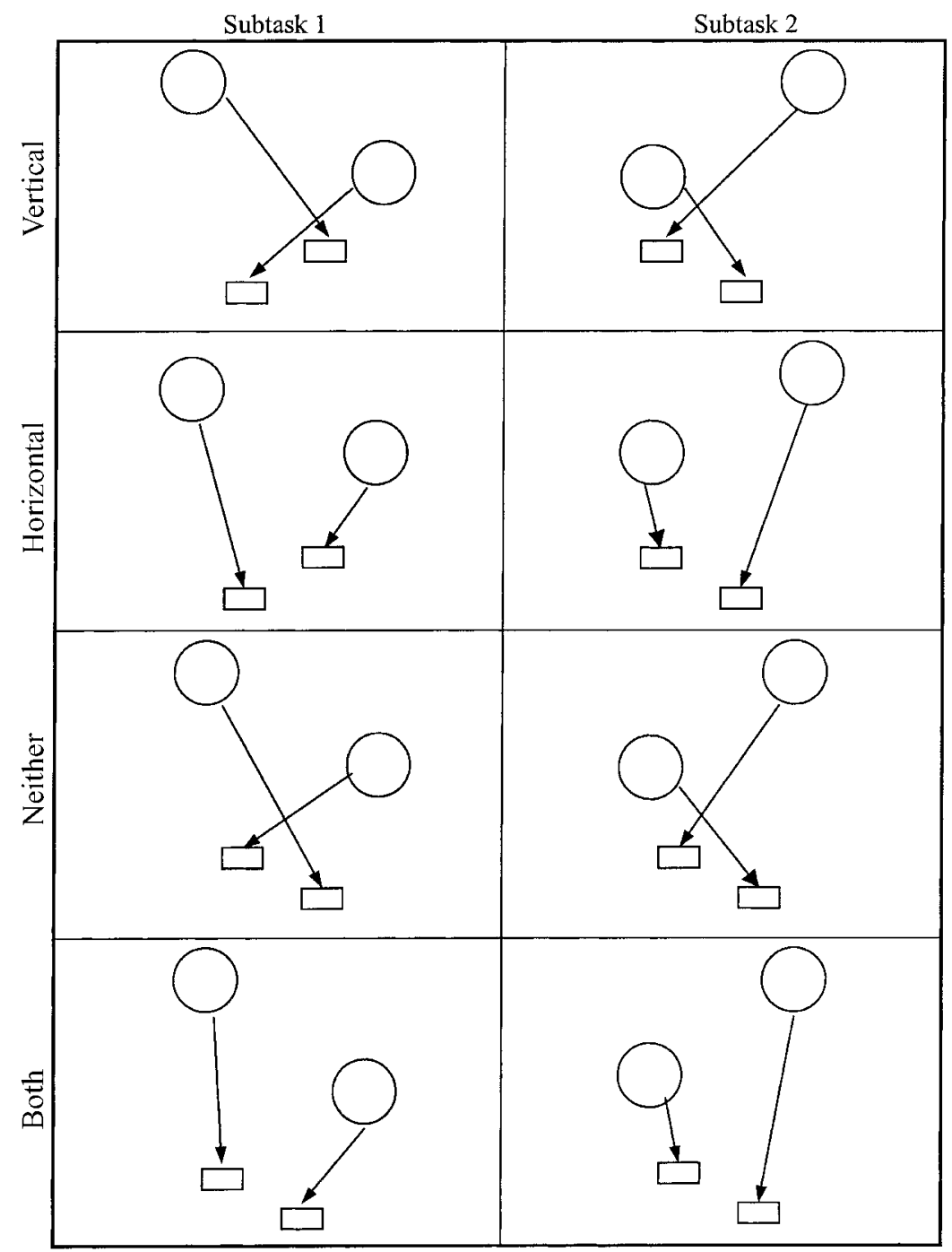

Figure 1. Illustration of the four stimulus-response compatibility conditions (vertical, horizontal, neither, and both) and subtasks. Stimulus locations are depicted by circles, and response locations by rectangles.

(Nicoletti \& Umiltà, 1985). Nicoletti and Umiltà also examined the relative magnitudes of the compatibility effects for the horizontal and the vertical dimensions. The compatibility effect for the horizontal dimension was obtained by subtracting the average of the conditions that were compatible along the horizontal dimension (horizontal and both conditions) from the average of the conditions that were incompatible along the horizontal dimension (neither and vertical conditions). The compatibility effect for the vertical dimension was obtained in an analogous manner. Nicoletti and Umiltà found that the horizontal compatibility effect was larger than the vertical compatibility effect, even though subjects were instructed to respond in terms of above and below locations, and not left and right ones. They called this finding right-left prevalence because horizontal compatibility was more dominant than vertical compatibility.

\section{Defining Right-Left Prevalence}

The finding of right-left prevalence in the studies of Nicoletti and Umiltà (1985; Nicoletti et al., 1988) was surprising because it was counter to their instructions, which stressed the vertical dimension. Hommel (1996) instructed one group of subjects in terms of the vertical dimension and another in terms of the horizontal dimension in order to evaluate how instructions modulate the magnitudes of the horizontal and vertical compatibility effects. He found that the horizontal compatibility effect was larger than the vertical compatibility effect with horizontal instructions, but not with vertical instructions. On the basis of these results, Hommel concluded that a right-left advantage is obtained only with horizontal instructions. He attributed Nicoletti and Umiltà's (1985) finding of right-left prevalence under vertical instructions to their subjects' not following the vertical instructions and responding on the 
basis of horizontal codes. However, Vu et al. (2000) noted that Hommel did find a form of right-left prevalence in his study in that the benefit of horizontal compatibility over vertical compatibility with horizontal instructions was larger than the benefit of vertical compatibility over horizontal compatibility with vertical instructions.

As in Hommel's (1996) study, an advantage is often obtained for the dimension to which instructions direct the subject's attention (Vu \& Proctor, 2001; Vu et al., 2000). However, use of the term prevalence has been restricted to advantages for one dimension or the other that cannot be attributed solely to the instructed dimension. Consequently, in this paper, we use the term advantage to refer to a benefit for the instructed dimension, but we do not equate this advantage with the prevalence effect. The prevalence effect will be defined in two ways. First, prevalence is obtained when, collapsed across the instructional conditions, the compatibility effect for one dimension is larger than that obtained along the other dimension, as in Hommel's study. For example, right-left prevalence would be evident if the benefit for horizontal compatibility over vertical compatibility with horizontal instructions was larger than the benefit of vertical compatibility over horizontal compatibility with vertical instructions. Second, prevalence is also evident when a larger compatibility effect is obtained for a specific dimension when the instructions emphasize the alternative dimension, as in Nicoletti and Umiltà's (1985) study. For example, right-left prevalence would be evident if the horizontal compatibility effect was larger than the vertical compatibility effect under vertical instructions.

\section{Explanations for Right-Left Prevalence}

Many explanations for the right-left prevalence effect have been suggested (see Vu \& Proctor, 2001, for a detailed discussion). Nicoletti and Umiltà (1984, 1985; Nicoletti et al., 1988) offered three hypotheses that took into account the effectors used for responding, coding of stimuli with respect to the midline of the body, and attentional allocation. However, they were not able to find evidence that strongly supported any one of the hypotheses. Hommel (1996) proposed a fourth hypothesis that attributes rightleft prevalence to a voluntary strategy of horizontal coding adopted by subjects. Furthermore, he reevaluated Nicoletti and Umiltà's effector-based account and provided more convincing evidence that the use of right-left effectors is a factor in producing the prevalence effect. Although these accounts were able to explain specific incidences of right-left prevalence, none of them provided an adequate explanation of all the conditions in which the right-left prevalence effect was obtained.

Many of the phenomena associated with right-left prevalence and its dependence on the use of right-left effectors can be explained within two general theoretical frameworks of S-R compatibility. The first explanation is derived from Heister, Schroeder-Heister, and Ehrenstein's (1990) hierarchical model of spatial SRC, which distinguishes three factors that affect SRC: spatial coding of response keys (compatibility owing to the coding of response keys' positions), spatial coding of effector position (compatibility owing to the coding of the position of the responding hand or finger), and spatio-anatomical mapping (compatibility owing to the coding of response effectors as right or left). According to Heister et al., these factors "can jointly determine an observed effect" (p. 132), with the effect contributed by each factor weighted according to its rank order. Thus, an effector-based explanation for right-left prevalence can be derived because the condition with horizontal compatibility benefits from all three factors, whereas the condition with vertical compatibility benefits from only the first two. This implies that rightleft prevalence is due to the presence of spatio-anatomical mapping for the horizontal dimension, but not for the vertical dimension.

The second theoretical account for right-left prevalence can be drawn from Proctor and Reeve's $(1985,1986)$ salient features coding principle. According to this principle, subjects code S-R sets with respect to their salient dimensions, and performance is best when the salient dimensions of the stimulus and the response sets correspond. Proctor and Reeve presented evidence that the codes used for S-R translation are a function of the salient features of the S-R sets. Reeve, Proctor, Weeks, and Dornier (1992) examined four-choice precuing tasks in which stimuli were arranged linearly along the horizontal dimension and showed that, by systematically altering the grouping of the stimulus set to enhance the salience of particular pairs, different precuing benefits were obtained. Beyak, Weeks, and Chua (2000) used the same S-R arrangements as Reeve et al. but varied the salience of the response set. Beyak et al. obtained results similar to those of Reeve et al., although the effects were weaker. Proctor and Reeve (1986) showed that manipulating the salience of stimulus pairs arrayed in the vertical dimension also yielded precuing benefits in favor of the pair that was more salient. In terms of salient features coding, right-left prevalence would be a result of the horizontal dimensions' being made more salient than the vertical dimension by the response environment (i.e., use of left and right hands).

\section{Evaluating the Hierarchical and the Salient Features Coding Accounts of Two-Dimensional SRC}

$\mathrm{Vu}$ and Proctor (2001) rejected an effector-based account derived from the hierarchical model for explaining right-left prevalence, because they did not obtain the right-left prevalence effect when subjects responded with two fingers of the same hand. If the use of right-left effectors is the critical factor for producing right-left prevalence, the effect should have been evident, because the two fingers can be regarded as right-left effectors and typically yield an SRC effect of similar magnitude to that of two fingers on different hands (Heister et al., 1990). In addition, $\mathrm{Vu}$ and Proctor showed that top-bottom prevalence was obtained when the response environment made the vertical dimension more salient by having subjects respond with ipsilateral hand and foot effectors or with one 
hand placed on top of the other. Furthermore, in Vu et al.'s (2000) experiments, the size of the right-left prevalence effect was larger when responses were made with the hands held widely apart on a handgrip apparatus than with the hands placed close together on a keypad. This outcome was probably due to the right-left distinction's being more salient with the former response environment than with the latter. Thus, $\mathrm{Vu}$ and Proctor concluded that prevalence is not a result of the effectors used for responding per se, but rather of coding based on the salient frame of reference provided by the response environment.

\section{Present Study}

Vu and Proctor (2001) were able to systematically alter the prevalence effect with manipulations of response configurations; however, they did not manipulate the salience of stimulus displays. Determining whether manipulation of salience with respect to the stimulus set can alter the relative benefits for the horizontal and the vertical dimensions is important because, according to Proctor and Reeve's $(1985,1986)$ salient features coding principle, the relative salience of both stimulus and response sets should influence coding.

The purpose of this study was to determine (1) whether manipulation of the salience of stimulus displays can systematically alter the magnitude of two-dimensional compatibility effects and (2) whether performance is best for a specific dimension (horizontal or vertical) when the salient features of the S-R sets correspond for that dimension. Experiment 1 was designed to examine whether a manipulation of salience with stimulus sets can systematically alter the prevalence effect. Responses were keypresses made with the index fingers of both hands, which produce the weaker form of right-left prevalence with equivalent stimulus displays. In Experiments 2 and 3, the correspondence between the S-R sets was examined. In Experiment 2, the horizontal-and vertical-salient stimulus displays used in Experiment 1 were mapped to keypress responses made with the index fingers of the left and right hands that were positioned to make either the horizontal or the vertical dimension more salient. In Experiment 3, the display manipulations were paired with ipsilateral or contralateral hand-foot responses. According to the salient features coding principle, the benefit of horizontal compatibility over vertical compatibility should be largest when the salient features of the $\mathrm{S}-\mathrm{R}$ sets correspond to the horizontal dimension. Conversely, the benefit of vertical compatibility over horizontal compatibility should be largest when the salient features of the S-R sets correspond to the vertical dimension.

\section{EXPERIMENT 1}

Experiment 1 was designed to evaluate whether manipulating the salience of the stimulus display systematically alters the prevalence effect obtained with two-dimensional compatibility. Three display manipulations were used: equivalent, vertical salient, and horizontal salient. Salience was operationally defined in terms of proximity. For the equivalent display, the distances between the stimuli were equivalent along the horizontal and the vertical dimensions. For horizontal-salient displays, the stimuli were further apart on the horizontal dimension than on the vertical one. For vertical-salient displays, the stimuli were further apart on the vertical dimension than on the horizontal one (see Figure 2). To confirm that the proximity manipulation was sufficient to make one dimension more salient than the other, an experiment was conducted in which subjects responded along one dimension to the vertical- and the horizontal-salient stimuli mixed together. Thirty-two subjects participated, 16 responding with top and bottom keys (the 8 and 2 keys on the numeric keypad) and 16 with left and right keys (the 6 and 4 keys). When the responses were arrayed in the vertical dimension, the subjects were significantly faster with the vertical-salient stimuli than with the horizontal-salient ones (mean difference $[M D]=$ $90 \mathrm{msec}$ ). However, when the responses were arrayed in the horizontal dimension, the subjects were significantly faster with the horizontal-salient stimuli than with the vertical-salient ones $(M D=41 \mathrm{msec})$. Thus, the proximity manipulation is sufficient in making one dimension more salient than the other.

\section{Method}

Subjects. Ninety-six undergraduate volunteers were recruited from Purdue University for partial credit toward their introductory psychology course requirement.

Apparatus and Stimuli. An IBM-compatible microcomputer was used to present stimuli and record the subjects' responses and RTs. Micro Experimental Laboratory (MEL Version 2.0) was used to program the experiment. The stimuli were displayed on a 14-in. VGA monitor, which was viewed by the subject from approximately $60 \mathrm{~cm}$. The stimuli were solid circles with a diameter of $15 \mathrm{~mm}$ (a visual angle of approximately $1.43^{\circ}$ ). A $4-\mathrm{mm}$ cross was placed in the center of the display screen to serve as a fixation point. The circles were presented diagonally (top-left and bottom-right or topright and bottom-left), approximately $107 \mathrm{~mm}\left(10.1^{\circ}\right)$ from the central fixation point (see Figure 2). For the equivalent display condition, circles were presented one at a time in locations at angles of $45^{\circ}$ (top right), $135^{\circ}$ (top left), $225^{\circ}$ (bottom left), or $315^{\circ}$ (bottom right) from the central fixation point. For the vertical-salient display condition, circles were presented one at a time in locations at angles of $75^{\circ}$ (top right), $105^{\circ}$ (top left), $255^{\circ}$ (bottom left), or $285^{\circ}$ (bottom right) from the central fixation point. For the horizontal-salient display condition, circles were presented one at a time in locations at angles of $15^{\circ}$ (top right), $165^{\circ}$ (top left), $195^{\circ}$ (bottom left), or $345^{\circ}$ (bottom right) from the central fixation point.

The subjects responded to each stimulus by pressing the $1,3,7$, or 9 key on the numeric pad of the keyboard. The keyboard was placed so that the numeric pad was aligned with the center of the screen and with the midline of the body. The index fingers of bothhands were placed on two of the four response keys (either the 7 and 3 keys or the 1 and 9 keys), with the left index finger placed on the leftmost and the right index finger on the rightmost of the two keys.

Procedure. The experiment was conducted in a quiet, well-lit room. All the subjects completed four S-R compatibility conditions, each consisting of two subtasks (see Figure 1). The four conditions can be characterized as a combination of horizontal compatibility (compatible or incompatible) and vertical compatibility (compatible or incompatible): The S-R mapping is compatible along (1) the vertical and horizontal dimensions for the both condition, (2) only 


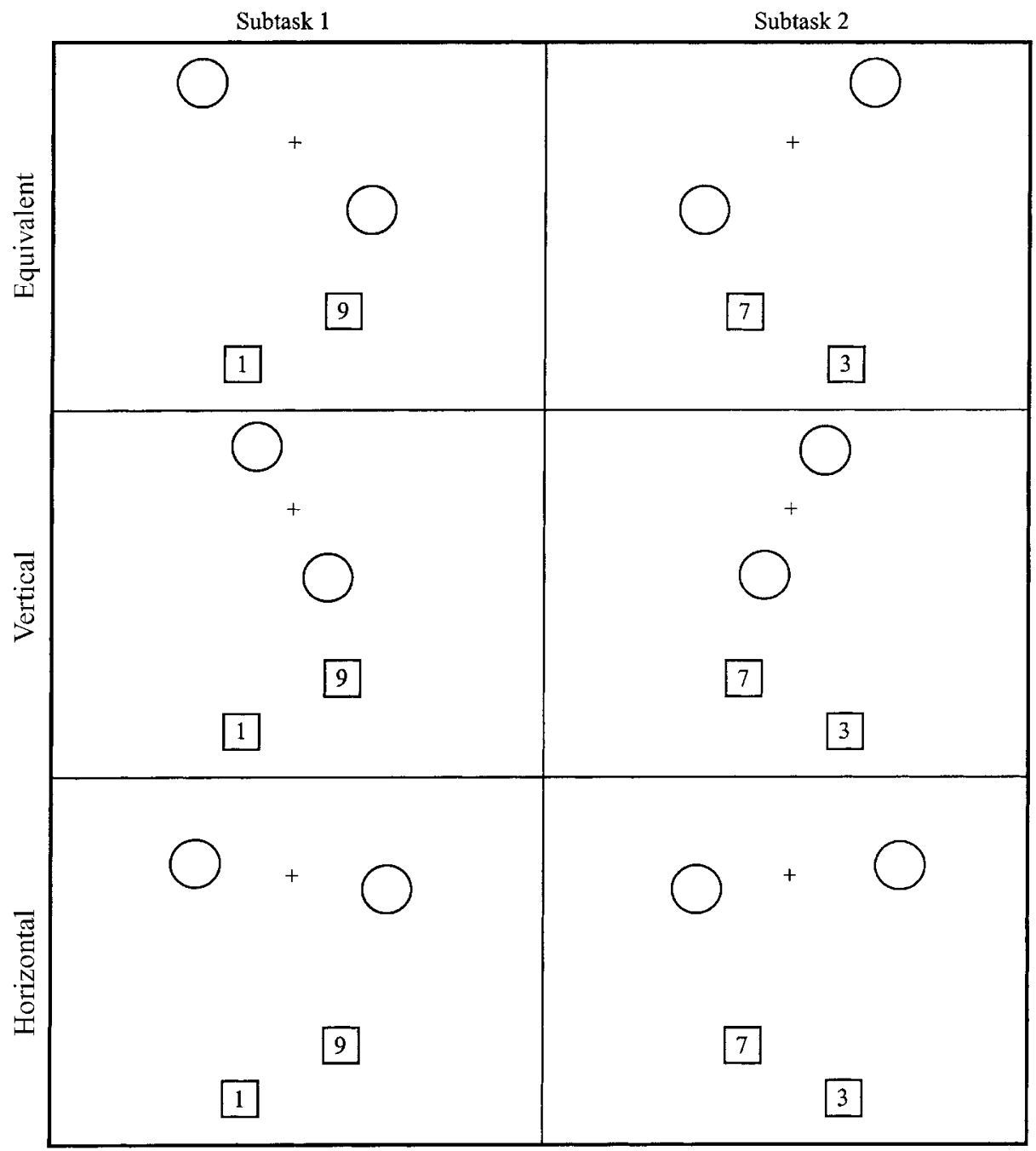

Figure 2. Illustration of the three salience conditions (equivalent, vertical salience, and horizontal salience) and their subtasks. Stimuli are depicted by circles, and response keys by squares.

the vertical dimension for the vertical condition, (3) only the horizontal dimension for the horizontal condition, and (4) neither dimension for the neither condition.

The subjects received the four compatibility conditions in different orders determined by a Latin square. The two subtasks within a condition differed in terms of the diagonal along which the two alternative stimuli and the two alternative responses were located, with those for Subtask 2 being the opposite of those for Subtask 1. The subjects completed both subtasks within a compatibility condition before receiving the next compatibility condition, and the order of the subtasks was counterbalanced between subjects, with half receiving Subtask 1 first and half receiving Subtask 2 first.

Thirty-two subjects were assigned to the equivalent display, 32 to the vertical-salient display, and 32 to the horizontal-salient display. Within each display condition, 16 subjects were given vertical instructions, and 16 were given horizontal instructions. For the vertical instructions group, the experimenter showed the subject where the cross (central fixation point) would be located and where the two stimuli would appear, referring to them as top circle and bottom circle. The experimenter also told the subject the appropriate response to each stimulus in terms of top-bottom locations (i.e., press the top key if the stimulus appears above the cross and press the bottom key if the stimulus appears below the cross, or vice versa). The words left and right were not used in the instructions. The other 16 subjects received the instructions in terms of horizontal dimensions.

Within each subtask of each condition, the subjects received a block of 10 practice trials followed by a block of 10 warm-up and 40 experimental trials. The practice and warm-up trials were not included in the data analyses. Therefore, each subject completed a total of eight experimental blocks, consisting of the two subtasks for each of the four S-R compatibility conditions. Each trial began with onset of the fixation cross, which was displayed for $1 \mathrm{sec}$. Simultaneous with the offset of the fixation cross, a circle appeared in one of the two locations and was displayed until a response was made. RT was measured from stimulus onset to the registration of a response. A 400$\mathrm{Hz}$ error tone was presented for $500 \mathrm{msec}$ for incorrect responses, followed by the 1-sec intertrial interval. The experimenter stayed in the room with the subject and repeated the instructions for each block before he or she began the practice trials for the block and made sure that the subject maintained the correct hand position. 
Table 1

Mean Reaction Time (RT; in Milliseconds) and Percentage of Errors (PE) for Compatibility Effects in Experiment 1 as a Function of Dimension, Instructions, and Salience

\begin{tabular}{|c|c|c|c|c|c|c|}
\hline \multirow[b]{3}{*}{ Instructions } & \multicolumn{4}{|c|}{ Dimensional Compatibility Effect } & & \\
\hline & \multicolumn{2}{|c|}{ Vertical $^{\mathrm{a}}$} & \multicolumn{2}{|c|}{ Horizontal $^{\mathrm{b}}$} & \multicolumn{2}{|c|}{ Difference ${ }^{\mathrm{c}, \mathrm{d}}$} \\
\hline & RT & PE & RT & $\mathrm{PE}$ & RT & $\mathrm{PE}$ \\
\hline \multicolumn{7}{|c|}{ Vertical-Salient Display } \\
\hline Vertical & 54 & 0.63 & 19 & 0.70 & -35 & 0.07 \\
\hline Horizontal & 5 & -0.01 & 35 & 1.40 & 30 & 1.41 \\
\hline Mean & 30 & 0.31 & 27 & 1.05 & -3 & 0.74 \\
\hline \multicolumn{7}{|c|}{ Equivalent Display } \\
\hline Vertical & 40 & 0.78 & 30 & -0.17 & -10 & -0.95 \\
\hline Horizontal & 9 & -0.04 & 57 & 1.44 & 48 & 1.48 \\
\hline Mean & 25 & 0.37 & 44 & 0.64 & 19 & 0.27 \\
\hline \multicolumn{7}{|c|}{ Horizontal-Salient Display } \\
\hline Vertical & 39 & 0.31 & 54 & 1.56 & 15 & 1.25 \\
\hline Horizontal & 0 & -0.23 & 60 & 0.71 & 60 & 0.94 \\
\hline Mean & 20 & 0.04 & 57 & 1.14 & 38 & 1.10 \\
\hline
\end{tabular}

${ }^{a}$ Standard error for vertical compatibility is 7.67 for RT and 0.34 for PE. ${ }^{\mathrm{b}}$ Standard error for horizontal compatibility is 8.37 for RT and 0.36 for PE. ${ }^{c}$ The difference is calculated by horizontal compatibility-vertical compatibility. ${ }^{\mathrm{d}}$ Standard error for the difference is 11.19 for RT and 0.50 for PE.

\section{Results}

RTs less than $100 \mathrm{msec}$ and greater than $1,000 \mathrm{msec}$ were not included in the analysis (fewer than $1 \%$ of all the trials). Mean RT and percentage of errors (PE) for each condition are shown in Appendix A. Because prevalence is defined with respect to the relative magnitudes of the horizontal and vertical compatibility effects, the reported analyses were performed on these effects (see Table 1). The compatibility effect for the horizontal dimension was obtained by subtracting the average of the conditions that were compatible along the horizontal dimension (horizon- tal and both conditions) from the average of the conditions that were incompatible along the horizontal dimension (neither and vertical conditions). The compatibility effect for the vertical dimension was obtained in an analogous manner.

Reaction time. Mean compatibility effects were submitted to 2 (compatibility: horizontal or vertical) $\times 2$ (instructions: horizontal or vertical) $\times 3$ (salience: horizontal, vertical, or equivalent) analyses of variance (ANOVAs). Overall, a right-left prevalence effect was obtained, with the horizontal compatibility effect $(M=43 \mathrm{msec})$ larger than the vertical compatibility effect $[M=25 \mathrm{msec}$; $\left.F(1,90)=15.20, M S_{\mathrm{e}}=1,002, p<.001\right]$. The magnitudes of the compatibility effects also varied as a function of instructions. With horizontal instructions, the horizontal compatibility effect was $46 \mathrm{msec}$ larger than the vertical compatibility effect, but with vertical instructions, the vertical compatibility effect was $10 \mathrm{msec}$ larger than the horizontal compatibility effect $[F(1,90)=37.5, p<.001]$.

Moreover, display salience also influenced the magnitudes of the horizontal and vertical compatibility effects $[F(2,90)=6.2, p=.003$; see Figure 3]. Similar to previous findings, right-left prevalence was obtained with the equivalent display, in which the horizontal compatibility effect $(M=44 \mathrm{msec})$ was larger than the vertical compatibility effect $(M=25 \mathrm{msec})$. With the horizontal-salient display, the right-left prevalence effect was enhanced: The horizontal compatibility effect $(M=57 \mathrm{msec})$ was larger than the vertical compatibility effect $(M=20 \mathrm{msec})$, and this right-left advantage was obtained regardless of instructions. With the vertical-salient display, however, the rightleft prevalence effect was eliminated, with the horizontal $(M=30 \mathrm{msec})$ and vertical $(M=27 \mathrm{msec})$ compatibility effects being of similar magnitude.

Percentage of error. Similar to the RT data, there was an overall right-left prevalence effect: The horizontal

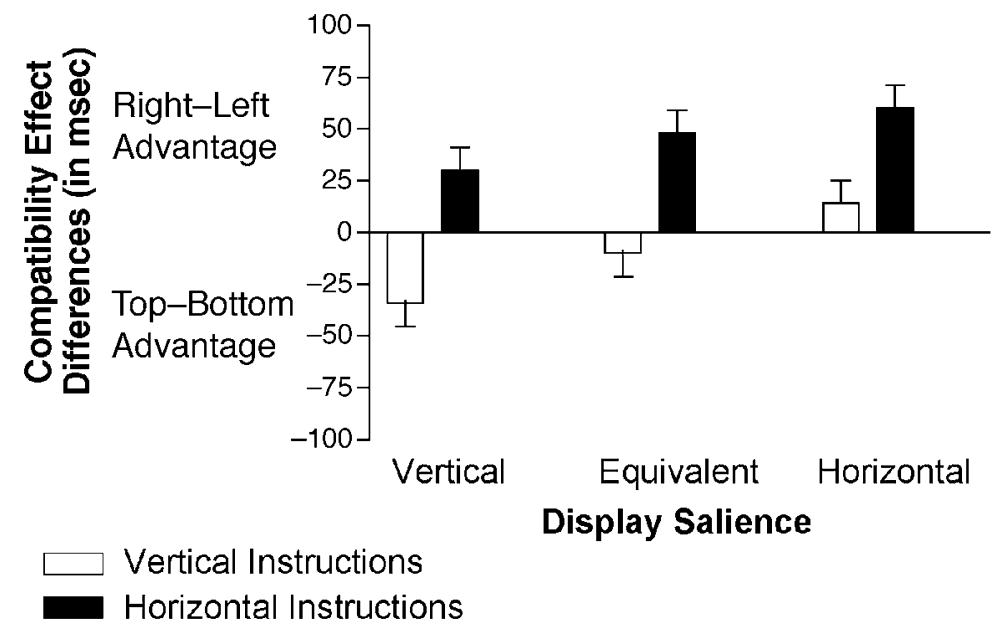

Figure 3. Mean compatibility effect differences (horizontal - vertical) in Experiment 1 as a function of display salience and instructions. 
compatibility effect $(M=0.94 \%)$ was larger than the vertical compatibility effect $[M=0.24 \% ; F(1,90)=11.93$, $\left.M S_{\mathrm{e}}=1.99, p=.001\right]$. However, the magnitudes of the compatibility effects were modified by a three-way interaction of compatibility with instructions and salience $[F(2,90)=7.58, p=.026]$. For equivalent and verticalsalient displays, right-left prevalence was evident in that the benefit for horizontal compatibility over vertical compatibility with horizontal instructions $(M D \mathrm{~s}=1.48 \%$ and $1.41 \%$, respectively) was larger than the benefit for vertical compatibility over horizontal compatibility with vertical instructions $(M D \mathrm{~s}=-0.95 \%$ and $-0.07 \%)$. For horizontalsalient displays, the stronger form of right-left prevalence was obtained in which the horizontal compatibility effect was larger than the vertical compatibility effect regardless of instructions $(M D=1.1 \%)$.

\section{Discussion}

The results of Experiment 1 show that manipulating the relative salience of display configurations systematically altered the magnitude of the prevalence effect obtained with two-dimensional compatibility. With the equivalent display, the weaker form of right-left prevalence was evident, in which right-left prevalence was obtained across instructions but was not strong enough to be apparent with vertical instructions alone, a finding that is similar to that of previous studies (Vu \& Proctor, 2001; Vu et al., 2000). With the horizontal-salient display, a stronger form of right-left prevalence effect was evident, in which the benefit for horizontal compatibility was enhanced, and a right-left benefit was obtained regardless of instructions. With the vertical-salient display, the right-left prevalence effect was eliminated for the RT data. Thus, Experiment 1 showed that manipulations of relative salience for stimulus display configurations alter the magnitude of the right-left prevalence effect.

The results of Experiment 1 and Vu and Proctor's (2001) study show that the magnitude of the right-left prevalence effect is affected by the relative salience of the horizontal and vertical dimensions for both the stimulus and the response sets, consistent with Proctor and Reeve's (1985, 1986) salient features coding principle. The asymmetric results in Experiment 1, in which an overall right-left prevalence was evident, are in agreement with the principle as well. Because responses were made with the left and the right hands placed on corresponding sides of the body, the response configuration favored the horizontal dimension (Vu et al., 2000). According to the salient features coding principle, S-R translation along a particular dimension should be fastest when the salient features of the $\mathrm{S}-\mathrm{R}$ sets correspond for that dimension. Because both the stimuli and the responses were salient on the horizontal dimension for the condition with horizontal-salient displays, this condition showed a strong right-left prevalence effect. However, because only the stimuli were salient on the vertical dimension for the condition with verticalsalient displays, this condition did not show a top-bottom prevalence effect.

\section{EXPERIMENT 2}

Experiment 2 was designed to evaluate the prediction of the salient features coding principle that when S-R sets vary along vertical and horizontal dimensions simultaneously, the benefit for a particular dimension will be largest when that dimension is salient for both the stimulus and the responses sets. For the stimulus manipulation, the horizontal-and the vertical-salient displays were identical to those of Experiment 1. For the response manipulation, the hands were positioned adjacent to each other for the horizontal-salient response condition and on top of each other for the vertical-salient response condition. These response arrangements were chosen because, with the equivalent display, right-left prevalence was obtained in the former case and top-bottom prevalence in the latter case ( $\mathrm{Vu}$ \& Proctor, 2001).

\section{Method}

Subjects. One hundred and twenty-eight new subjects were recruited from the same pool as that in Experiment 1. Thirty-two subjects were assigned to the horizontal-salient-stimulus/horizontalsalient-response condition, 32 to the horizontal-salient-stimulus/ vertical-salient-response condition, 32 to the vertical-salient stimulus/horizontal-salient-response condition, and the 32 to the vertical-salient-stimulus/vertical-salient-response condition. Within each salience condition, 16 were instructed in terms of top-bottom locations, and 16 were instructed in terms of right-left locations.

Apparatus, Stimuli, and Procedure. The apparatus, stimuli, and procedure were identical to those used in Experiment 1, except for the differences noted. As in Experiment 1, the horizontalsalient-stimulus/horizontal-salient-response condition used the horizontal-salient display and keypress responses with the hands placed adjacent to each other. For the horizontal-salient-stimulus/ vertical-salient-response condition, responses were also made with keypresses, but the subjects placed one hand on top of the other. The top hand was used to make the top response, and the bottom hand was used to make the bottom response; however, the right index finger was used to make the left response, and left index finger was used to make the right response. For example, if responses were made with the 7 and 3 keys, the left index finger was placed on the $3 \mathrm{key}$, and the right hand was placed on top of the left hand, with the right index finger on the 7 key. For the vertical-salient-stimulus/horizontalsalient-response and the vertical-salient-stimulus/ vertical-salientresponse conditions, the stimuli were identical to the vertical-salient display in Experiment 1, and the response configurations were the same as those used for the horizontal/vertical-salient pairings.

\section{Results}

Similar analyses to those of Experiment 1 were performed on the RT and PE data, with the difference being that display salience had only two levels (horizontal and vertical), and response salience (horizontal or vertical) was an additional factor. The compatibility effects for the horizontal and vertical dimensions are shown in Table 2, the differences in these two effects in Figure 4, and the means for the individual conditions in Appendix B.

Reaction time. There was an overall top-bottom prevalence in that the vertical compatibility effect $(M=43 \mathrm{msec})$ was larger than the horizontal compatibility effect $[M=$ $\left.33 \mathrm{msec} ; F(1,120)=4.2, M S_{\mathrm{e}}=1,427, p=.044\right]$. Compatibility also entered into two-way interactions with instruc- 
Table 2

Mean Reaction Time (RT; in Milliseconds) and Percentage of Errors (PE) for Compatibility Effects in Experiment 2 as a Function of Dimension, Instructions, and Display-Response Salience

Dimensional Compatibility Effect

\begin{tabular}{|c|c|c|c|c|c|c|}
\hline \multirow[b]{3}{*}{ Instructions } & \multicolumn{6}{|c|}{ Dimensional Compatibility Effect } \\
\hline & & & & $\mathrm{tal}^{\mathrm{b}}$ & & \\
\hline & RT & PE & RT & PE & RT & $\mathrm{PE}$ \\
\hline
\end{tabular}

\begin{tabular}{lcccccr}
\hline \multicolumn{7}{c}{ Vertical-Salient Display-Vertical-Salient Responses } \\
Vertical & 75 & 0.56 & -16 & -0.32 & -91 & -0.88 \\
Horizontal & 48 & 0.00 & 31 & 0.70 & -17 & 0.70 \\
Mean & 62 & 0.28 & 8 & 0.19 & -54 & -0.09 \\
\multicolumn{5}{c}{ Vertical-Salient } & Display-Horizontal-Salient Responses \\
Vertical & 68 & 0.78 & 8 & 0.31 & -60 & -0.47 \\
Horizontal & 15 & 0.35 & 57 & 0.90 & 42 & 0.55 \\
Mean & 42 & 0.57 & 33 & 0.61 & -9 & 0.04 \\
\multicolumn{7}{c}{ Horizontal-Salient Display-Vertical-Salient Responses } \\
Vertical & 62 & 0.70 & 25 & 0.08 & -37 & -0.62 \\
Horizontal & 26 & 1.33 & 42 & 0.86 & 16 & -0.47 \\
Mean & 44 & 1.02 & 34 & 0.47 & -11 & -0.55 \\
$\quad$ Horizontal-Salient Display-Horizontal-Salient Responses \\
Vertical & 29 & 0.36 & 53 & 0.66 & 24 & 0.30 \\
Horizontal & 15 & -0.07 & 61 & 1.71 & 46 & 1.78 \\
Mean & 22 & 0.15 & 57 & 1.19 & 35 & 1.04 \\
\hline
\end{tabular}

a Standard error for vertical compatibility for RT and PE is 9.74 and 0.31 . ${ }^{\mathrm{b}}$ Standard error for horizontal compatibility for RT and PE is 9.48 and 0.31 . ${ }^{\mathrm{c}}$ The difference is calculated by horizontal compatibility-vertical compatibility. ${ }^{\mathrm{d}}$ Standard error for the difference for RT and PE is 13.36 and 0.51 .

tions, display salience, and response salience $[F \mathrm{~s}(1,120)>$ $20.66, p s<.001]$. The horizontal compatibility effect was $22 \mathrm{msec}$ larger than the vertical compatibility effect with horizontal instructions, and the vertical compatibility effect was $41 \mathrm{msec}$ larger than the horizontal compatibility effect with vertical instructions. Furthermore, top-bottom prevalence, in which the vertical compatibility effect was larger than the horizontal compatibility effect, was obtained with vertical-salient displays $(M D=32 \mathrm{msec})$ and vertical-salient responses $(M D=33 \mathrm{msec})$. Similarly, right-left prevalence, in which the horizontal compatibility effect was larger than the vertical compatibility effect, was obtained with horizontal-salient displays $(M D=$ $12 \mathrm{msec}$ ) and horizontal-salient responses $(M D=$ $13 \mathrm{msec}$ ). Display salience, instructions, and compatibility also interacted $[F(1,120)=7.39, p=.008]$. The topbottom advantage obtained with vertical instructions was larger with vertical-salient displays $(M D=76 \mathrm{msec})$ than with horizontal-salient displays $(M D=7 \mathrm{msec})$, and the right-left advantage obtained with horizontal instructions was larger with horizontal-salientdisplays $(M D=31 \mathrm{msec})$ than with vertical-salient displays $(M D=13 \mathrm{msec})$. In other words, there was little effect of stimulus salience with horizontal instructions, but a large effect of it with vertical instructions.

Percentage of error. Unlike the RT data, there was no overall prevalence effect, but the horizontal and vertical compatibility effects were modified by two-way interactions with response salience and instructions $\left[F_{\mathrm{s}}(1,120)<\right.$ $\left.5.64, M S_{\mathrm{e}}=2.12, p \mathrm{~s}<.019\right]$. The vertical compatibility effect was larger than the horizontal compatibility effect with vertical-salient responses $(M D=0.32 \%)$ and vertical instructions $(M D=0.42 \%)$. However, the horizontal compatibility effect was larger than the vertical compatibility effect with horizontal-salient responses $(M D=0.54 \%)$ and horizontal instructions $(M D=0.64 \%)$. Compatibility also entered into a three-way interaction with display salience and response salience $[F(1,120)=4.12, p=.045]$. The vertical compatibility effect was larger than the horizontal compatibility effect with vertical-salient responses, and this difference was nonsignificantly larger with horizontal-salientdisplays $(M=0.55 \%)$ than with vertical-

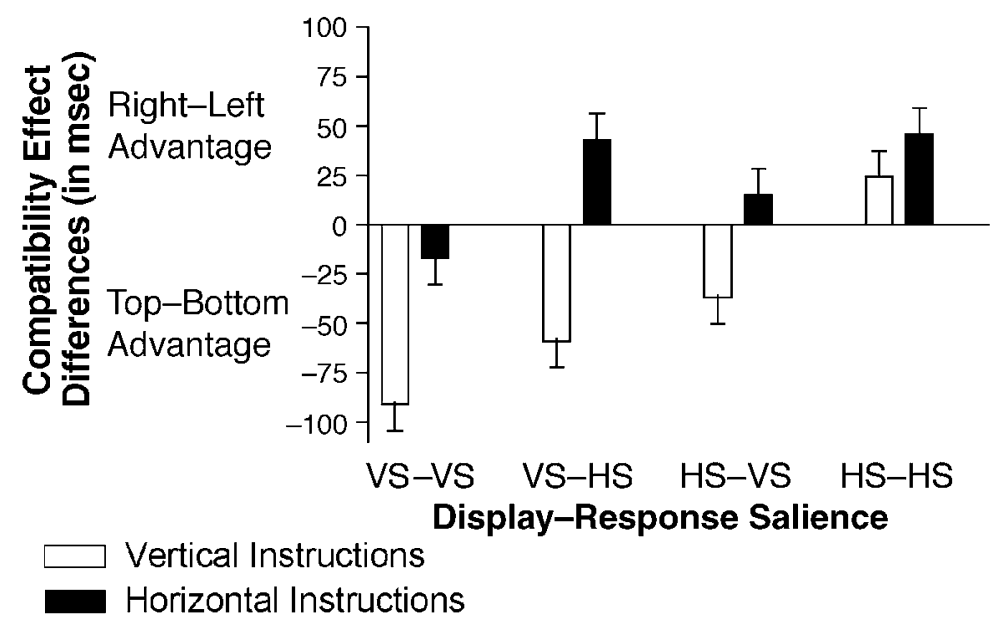

Figure 4. Mean compatibility effect differences (horizontal - vertical) in Experiment 2 as a function of display-salience-response-salience and instructions. VS is vertical salience, HS is horizontal salience, and the display salience is listed prior to the response salience. 
salient displays $(M=0.09 \%)$. The horizontal compatibility effect was larger than the vertical one with horizontal instructions, and this difference was larger with horizontalsalient displays $(M=1.05 \%)$ than with vertical-salient displays $(M=0.04 \%)$.

\section{Discussion}

Experiment 2 replicated the results of Experiment 1 and, together with Vu and Proctor's (2001) Experiment 4, shows that manipulations of salience for display and response configurations systematically alter the magnitude of the prevalence effect. The horizontal compatibility effect was larger than the vertical compatibility effect when the horizontal dimension was made more salient than the vertical dimension by the stimulus display or response configuration. Similarly, the vertical compatibility effect was larger than the horizontal compatibility effect when the vertical dimension was made more salient than the horizontal dimension by the stimulus display or response configuration. Furthermore, a right-left advantage was obtained regardless of instructions when both S-R sets emphasized the horizontal dimension, and a top-bottom advantage was obtained regardless of instructions when both S-R sets emphasized the vertical dimension (see Figure 4). These results are consistent with Proctor and Reeve's $(1985,1986)$ salient features coding principle, according to which performance is a function of the salience of S-R sets.

The differences between the horizontal and the vertical compatibility effects are shown in the right column of Table 2 . The benefit for horizontal compatibility over vertical compatibility (as indicated by positive values) is largest when the salient dimensions of both the display and the response configurations are made to favor the horizontal dimension, in comparison to when only one of the two made the horizontal dimension more salient. In addition, the benefit for vertical compatibility over horizontal compatibility (as indicated by negative values) is present when the salient dimensions of both the display and the response configurations are made to favor the vertical dimension. The compatibility effects of the conditions in which the salient features of the display and response do not correspond ( -9 and $-11 \mathrm{msec}$ ) are smaller than the effects of the conditions in which the salient features of the vertical $(-54 \mathrm{msec})$ or the horizontal $(35 \mathrm{msec})$ dimensions correspond. Thus, the results show that $\mathrm{S}-\mathrm{R}$ translation along either the vertical or the horizontal dimension was fastest when the salient dimensions of the display and the response configurations corresponded for that dimension. Furthermore, the nonsignificant interaction of display salience and response salience indicates that the correspondence benefits for the horizontal and the vertical dimensions are of similar magnitude.

\section{EXPERIMENT 3}

The results of Experiment 2 showed a salience correspondence effect: The benefit of horizontal compatibility over vertical compatibility was greatest when the salient dimensions of the S-R sets corresponded to the horizontal dimension, and the benefit of vertical compatibilityover horizontal compatibility was greatest when the salient dimensions of the S-R sets corresponded to the vertical dimension. However, the top-bottom hand placement of Experiment 2 resulted in significantly slower responses than did the adjacent hand placement $[M D=34 \mathrm{msec} ; F(1,120)=$ $19.12, p<.001$; see Appendix B], and thus S-R translation may have been affected by the "unnatural" nature of the top-bottom hand placement. Experiment 3 was designed to test the salience correspondence effect by using top-bottom effectors that can be naturally positioned. If the pattern of correspondence results is similar to that of Experiment 2, the correspondence effect obtained with two-dimensional S-R sets is reliable and generalizable to other response modes.

$\mathrm{Vu}$ and Proctor (2001) showed that the right-left prevalence effect could be obtained when top-bottom effectors are used for responding. More specifically, Vu and Proctor showed that right-left prevalence was obtained with contralateral hand-foot responses because the effectors can be coded easily along the horizontal dimension and that topbottom prevalence was obtained with ipsilateral handfoot responses because there is no anatomical frame of reference for the horizontal dimension but there is one for the vertical dimension. Experiment 3 was designed to evaluate the effects of salience as a function of correspondence between S-R sets with responses made by top-bottom effectors. It was hypothesized that Experiment 3 would yield a pattern of results similar to that of Experiment 2.

\section{Method}

Subjects. One hundred and twenty-eight new subjects were recruited from the same pool as in Experiments 1 and 2. Thirty-two subjects were assigned to the horizontal-salient-stimulus/ horizontalsalient-response condition, 32 to the horizontal-salient-stimulus/ vertical-salient-response condition, 32 to the vertical-salient stimulus/horizontal-salient-response condition, and the 32 to the vertical-salient-stimulus/vertical-salient- response condition. Within each salience condition, 16 were instructed in terms of top-bottom locations, and 16 were instructed in terms of right-left locations.

Apparatus, Stimuli, and Procedure. The apparatus, stimuli, and procedure were identical to those used in Experiment 2, except for the differences noted. The subjects responded to each stimulus by pressing a button on a hand-held bicycle grip or stepping on a Treadlite II foot pedal. Both the handgrip and the foot pedal were connected to the MEL Serial Response Box (Model 200A). Each subject held the handgrip in the palm of his or her hand, with the thumb horizontally oriented, and used his or her thumb to press the microswitch. The subject placed his or her hand on the tabletop, with the handgrip held approximately $30 \mathrm{~cm}$ away from the computer screen, $15 \mathrm{~cm}$ to the right of central fixation for the right response and $15 \mathrm{~cm}$ to the left of fixation for the left response. The foot pedals were placed on the floor, approximately $74 \mathrm{~cm}$ directly below the positions of the handgrips.

For the contralateral effectors condition, the subject used his or her right or left hand to make the top response and the opposite-side foot to make the bottom response. The subject extended his or her arm perpendicularly from his or her side and held the handgrip directly in front of the body. The opposite foot was placed on the foot 
Table 3

Mean Reaction Time (RT; in Milliseconds) and Percentage of Errors (PE) for Compatibility Effects in Experiment 3 as a Function of Dimension, Instructions, and Display-Response Salience

Dimensional Compatibility Effect

\begin{tabular}{|c|c|c|c|c|c|c|}
\hline & \multirow{2}{*}{\multicolumn{2}{|c|}{ Vertical $^{a}$}} & \multirow{2}{*}{\multicolumn{2}{|c|}{ Horizontal $^{\mathrm{b}}$}} & \multirow{2}{*}{\multicolumn{2}{|c|}{ Difference ${ }^{\mathrm{c}, \mathrm{d}}$}} \\
\hline \multirow[b]{2}{*}{ Instructions } & & & & & & \\
\hline & RT & PE & RT & PE & RT & PE \\
\hline
\end{tabular}

\begin{tabular}{lcccccr}
\hline \multicolumn{7}{c}{ Vertical-Salient } \\
Vertical & 78 & 1.53 & 28 & 1.13 & -50 & -0.40 \\
Horizontal & 61 & 0.55 & 44 & 1.65 & -17 & 1.10 \\
$\quad$ Mean & 70 & 1.04 & 36 & 1.39 & -34 & 0.35 \\
\multicolumn{5}{c}{ Vertical-Salient } & Display-Horizontal-Salient Responses \\
Vertical & 62 & 1.53 & 28 & 0.89 & -34 & -0.64 \\
Horizontal & 20 & 0.04 & 64 & 1.37 & 44 & 1.33 \\
$\quad$ Mean & 41 & 0.79 & 46 & 1.13 & 5 & 0.35
\end{tabular}

Horizontal-Salient Display-Vertical-Salient Responses

$\begin{array}{lrrrrrr}\text { Vertical } & 60 & 1.09 & 40 & 0.94 & -20 & -0.15 \\ \text { Horizontal } & 29 & 0.55 & 56 & 1.33 & 27 & 0.78 \\ \quad \text { Mean } & 45 & 0.82 & 48 & 1.14 & 4 & 0.32\end{array}$

Horizontal-Salient Display-Horizontal-Salient Responses

\begin{tabular}{lrrrrrr} 
Vertical & 40 & 0.70 & 58 & 1.10 & 18 & 0.40 \\
Horizontal & 17 & -0.16 & 71 & 1.32 & 54 & 1.48 \\
Mean & 29 & 0.27 & 65 & 1.21 & 36 & 0.94 \\
\hline
\end{tabular}

a Standard error for vertical compatibility for RT and PE is 10.30 and 0.41 . b Standard error for horizontal compatibility for RT and PE is 9.36 and 0.38 . ${ }^{\mathrm{c}}$ The difference is calculated by horizontal compatibility-vertical compatibility. ${ }^{\mathrm{d}}$ Standard error for the difference for RT and PE is -12.40 and 0.50 .

pedal, with the foot positioned at a $45^{\circ}$ angle. For the ipsilateral effectors condition, the subject also used his or her right or left hand to make the top response, but used the same-side foot to make the bottom response. The leg was crossed over to the opposite side of the hand position and placed at a $45^{\circ}$ angle on the foot pedal.

\section{Results}

Reaction time. Trials with RTs less than $100 \mathrm{msec}$ or greater than $1,500 \mathrm{msec}$ (fewer than $1 \%$ ) were not included. The upper limit was increased because RTs tend to be slower with the foot than with the hand. Similar analyses to those of Experiment 2 were conducted. See Table 3 for the horizontal and vertical compatibility effects, Figure 5 for the differences in these two effects, and Appendix C for the means for each condition.

The factor of compatibility (horizontal, vertical) entered into two-way interactions with instructions, display salience, and response salience $\left[F_{\mathrm{s}}(1,120)<14.72, M S_{\mathrm{e}}=\right.$ $1,230, p s<.001]$. With horizontal instructions, the horizontal compatibility effect was $27 \mathrm{msec}$ larger than the vertical compatibility effect, but with vertical instructions, the vertical compatibility effect was $22 \mathrm{msec}$ larger than the horizontal compatibility effect. Top-bottom prevalence, in which the vertical compatibility effect was larger than the horizontal compatibility effect, was obtained with vertical-salient displays $(M D=15 \mathrm{msec})$ and verticalsalient responses $(M D=15 \mathrm{msec})$. Right-left prevalence, in which the horizontal compatibility effect was larger than the vertical compatibility effect, was obtained with horizontal-salient displays $(M D=20 \mathrm{msec})$ and horizontalsalient responses $(M D=21 \mathrm{msec})$.

Percentage of error. An overall right-left prevalence effect was obtained: The horizontal compatibility effect $(M=1.22 \%)$ was larger than the vertical compatibility effect $\left[M=0.73 \% ; F(1,120)=7.40, M S_{\mathrm{e}}=2.06, p \mathrm{~s}<.001\right]$. In addition, the horizontal and vertical compatibility effects were modified by instructions $[F(1,120)=14.59, p<$ $.001]$. With vertical instructions, the vertical compatibility effect was larger than the horizontal compatibility effect $(M D=0.20 \%)$, and with horizontal instructions, the

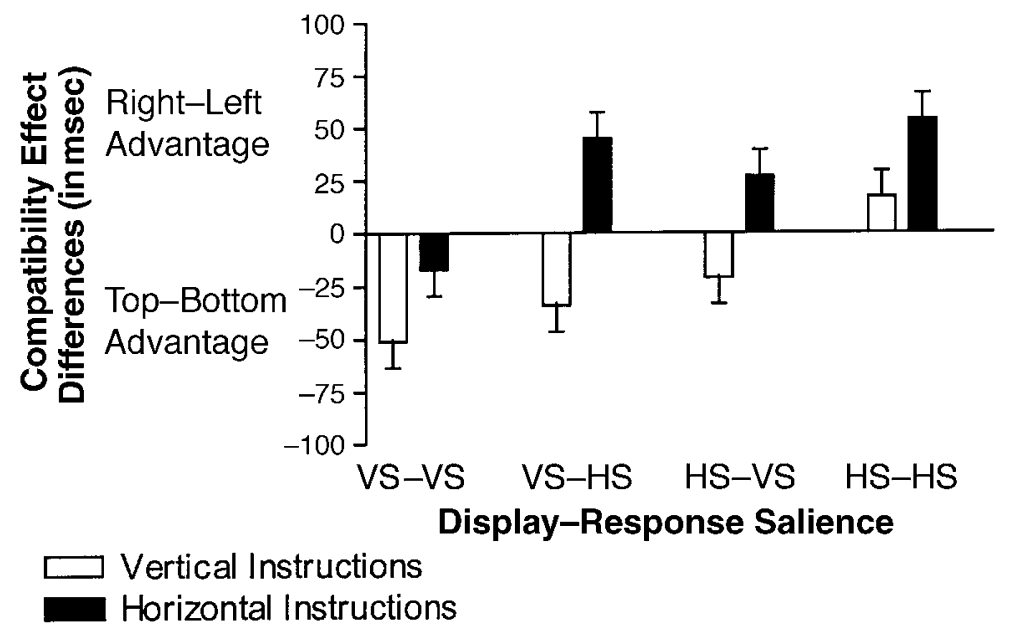

Figure 5. Mean compatibility effect differences (horizontal - vertical) in Experiment 3 as a function of display-salience-response-salience and instructions. VS is vertical salience, HS is horizontal salience, and the display salience is listed prior to the response salience. 
horizontal compatibility effect was larger than the vertical compatibility effect $(M D=1.17 \%)$.

\section{Discussion}

The mean RTs for ipsilateral and contralateral handfoot placements were similar in this experiment (see Appendix C), eliminating the possibility that slower response selection for one response configuration altered the pattern of S-R translation. Similar to Experiment 2, Experiment 3 showed that the benefit for horizontal compatibility over vertical compatibility was larger when the horizontal dimension was made more salient than the vertical dimension by the stimulus display or response configuration. Furthermore, the benefit for vertical compatibility over horizontal compatibility was larger when the vertical dimension was made more salient than the horizontal dimension by the stimulus display or response configuration.

The benefit of horizontal over vertical compatibility, or vice versa, similar to that reported in Experiment 2, showed that there was a correspondence effect in favor of the dimension made salient by both display and response configurations (see Table 3, last column). The benefit for horizontal compatibility over vertical compatibility (as indicated by the positive value) was largest when the salient dimensions of both the display and the response configurations favored the horizontal dimension, in comparison with the conditions in which only one of the two made the horizontal dimension more salient. In addition, the benefit for vertical compatibility over horizontal compatibility (as indicated by the negative values) was largest when the salient dimensions of both the display and the response configurations favored the vertical dimension than when only the response or display was made to favor the vertical dimension. The effects of the conditions in which the salient features of the display and response did not correspond (5 and $4 \mathrm{msec}$ ) were smaller than the effects of the conditions in which the salient features of the horizontal $(36 \mathrm{msec})$ or the vertical $(-34 \mathrm{msec})$ dimensions corresponded.

Again, the lack of a significant interaction of response salience and display salience can be attributed to the fact that the benefit of the display and response correspondence over noncorrespondence for the vertical and horizontal dimensions is of similar magnitude and going in opposing directions (in this case, negative when responding favors the vertical dimension and positive when favoring the horizontal dimension). Thus, taken together, the results of Experiments 2 and 3 are in close agreement, and both show that the benefit for the horizontal or the vertical dimension is largest when the salient features of the stimulus display and the response configuration correspond for that dimension.

\section{GENERAL DISCUSSION}

When S-R sets vary along the horizontal and the vertical dimensions simultaneously, a prevalence effect is often obtained in which one dimension is more dominant than the other (Vu \& Proctor, 2001). Right-left prevalence, the form of dominance that is usually obtained, has been shown to be evident in a variety of situations using different S-R modalities. Vu and Proctor showed that left-right effectors were important in producing right-left prevalence because they enhanced the salience of the horizontal dimension relative to the vertical one, possibly because the effectors provide a frame of reference for horizontal coding. However, even when responses are made with the left and right hands, top-bottom prevalence can be obtained if the hands are positioned on top of each other to make the vertical dimension more salient than the horizontal one. On the basis of the results of their study, Vu and Proctor proposed an account for the prevalence effect in terms of salient features coding (Proctor \& Reeve, 1985, 1986), in which prevalence is a result of subjects' coding responses on the basis of the dimension that is made salient by the response set. This account was the first one that was able to explain and predict the type and magnitude of prevalence effects obtained to date.

Because the salient features coding account of the right-left prevalence effect is the only explanation that is consistent with previous results obtained for twodimensional compatibility, we tested its specific implications. Experiment 1 manipulated the relative salience of stimulus displays by varying the proximity of the stimuli along the horizontal and vertical dimensions. It demonstrated that the manipulation of relative salience of stimulus displays alters the pattern of prevalence obtained. That is, right-left prevalence was enhanced with horizontalsalient displays and eliminated with vertical-salient displays. Taken together, the results of Experiment 1 and $\mathrm{Vu}$ and Proctor's (2001) study show that manipulations of the relative salience of stimulus displays or response configurations can systematically alter the type of prevalence effect obtained, and these results support salient features coding as a valid explanation for the prevalence effect.

Proctor and Reeve's $(1985,1986)$ salient features coding principle emphasizes that $\mathrm{S}-\mathrm{R}$ translation for a specific dimension should be fastest when the salient features of the display and response configurations correspond to that dimension. The results of both Experiment 2, in which responses were made with the hands placed in the topbottom or adjacent hand placement, and Experiment 3, in which the responses were made with the contralateral and ipsilateral hand-foot placement, show similar patterns of results that are in accordance with this principle. The benefits of horizontal and vertical compatibility were larger when the salient features of the S-R sets corresponded to the specific dimension than when they did not. In particular, RTs were faster for the condition with horizontal compatibility alone than for the condition with vertical compatibility alone when the salient features of both the display and the response configuration made the horizontal dimension more salient. With vertically salient display and response correspondence, the reverse pattern of results 
was obtained: Performance was better on the condition with vertical compatibility alone than in the condition with horizontal compatibility alone.

When the salient dimension of the stimulus display did not correspond to the salient dimension of the response configuration, the conditions with horizontal and vertical compatibility alone were similar. In Experiment 2, the noncorresponding S-R sets tended to favor the vertical dimension $(M D=10 \mathrm{msec})$, whereas in Experiment 3 they tended to favor the horizontal dimension $(M D=5 \mathrm{msec})$, although these differences were not statistically significant. The results are in general agreement with the precuing effects obtained by Reeve et al. (1992), in which manipulating the salient features of the stimulus display systematically altered the pattern of precuing benefits and performance was better when the salient features of the S-R sets corresponded than when they did not. Beyak et al. (2000) showed that manipulating the salient features of response configurations also yielded an effect, although the effect was much weaker, being significant mainly in the error data. Thus, unlike the precuing effects, which show a stronger effect of stimulus display salience, manipulations of salience for two-dimensional compatibility show equivalent benefits of the horizontal and vertical compatibility with display and response sets.

In previous studies that used the equivalent display (Hommel, 1996; Vu \& Proctor, 2001; Vu et al., 2000), instructions influenced the relative magnitudes of the compatibility effects. Because this display does not provide a salient frame of reference to influence coding with respect to one dimension over the other, there was an advantage for whichever dimension was emphasized by the instructions. However, this advantage was larger when the instructed dimension was also consistent with the dimension made salient by the response set ( $\mathrm{Vu} \&$ Proctor, 2001). In the present experiments, we found a similar influence of instructions: With horizontal instructions, a right-left advantage was obtained, and with vertical instructions, a top-bottom advantage was obtained. When the salient features of the S-R sets did not correspond, any prevalence effect was evident only when collapsed across instructions. However, when the salient features of the S-R corresponded to favor a single dimension, there was a benefit for compatibility on the salient dimension regardless of instructions. This benefit was smaller when the instructions were in terms of the nonsalient dimension than when it was in terms of the salient dimension. That an advantage for the dimension made salient for the display and response configurations is obtained even when instructions emphasize the other dimension reflects the strength of the frame of reference provided by the display and response organization.

The finding of right-left prevalence is considered to be of theoretical importance because it suggests that there may be a capacity limitation for processing more than one dimension. The robustness of right-left prevalence in studies of Nicoletti and Umiltà (1984, 1985; Nicoletti et al., 1988) led them to conclude that when both horizontal and vertical codes are present simultaneously, the ability to code vertical locations is reduced or eliminated. The present study, along with those of Vu et al. (2000) and Vu and Proctor (2001), indicates that right-left prevalence is not a result of a capacity limitation that allows for the coding of only one dimension or of horizontal codes automatically dominating vertical codes. Right-left prevalence is usually obtained over top-bottom prevalence because most response environments have subjects respond with left and right hands, thus providing a salient frame of reference for horizontal coding.

In summary, when S-R sets vary along horizontal and vertical dimensions simultaneously, both horizontal and vertical dimensions can be coded. The prevalence of one dimension over the other is a result of coding with respect to the dimension made salient by either the stimulus display or the response configuration. Furthermore, the benefits of both horizontal and vertical compatibility are greatest when the salient features of the S-R sets correspond on that dimension than when they do not. Overall, the results indicate that salient features coding provides a good explanation of the prevalence effect obtained in twodimensional compatibility.

\section{REFERENCES}

Beyak, B., Weeks, D., \& ChuA, R. (2000). Salience of responsefeatures in the spatial precuing task. Unpublished manuscript.

Heister, G., Schroeder-Heister, P., \& Ehrenstein, W. H. (1990). Spatial coding and spatio-anatomical mapping: Evidence for a hierarchical model of spatial stimulus-response compatibility. In R. W. Proctor \& T. G. Reeve (Eds.), Stimulus-response compatibility: An integrated perspective (pp. 117-143). Amsterdam: North-Holland.

Hommel, B. (1996). No prevalence of right-left over top-bottom spatial codes. Perception \& Psychophysics, 58, 102-110.

Hommel, B., \& Prinz, W. (Eds.) (1997). Theoretical issues in stimulusresponse compatibility. Amsterdam: North-Holland.

Nicoletti, R., \& Umiltà, C. (1984). Right-left prevalence in spatial compatibility. Perception \& Psychophysics, 35, 333-343.

Nicoletti, R., \& Umiltà, C. (1985). Responding with hand and foot: The right/left prevalence in spatial compatibility is still present. Perception \& Psychophysics, 38, 211-216.

Nicoletti, R., Umiltà, C., Tressoldi, E. P., \& Marzi, C. A. (1988). Why are left-right spatial codes easier to form than above-below ones? Perception \& Psychophysics, 43, 287-292.

Proctor, R. W., \& Dutta, A. (1993). Do the same stimulus-response relations influence choice reactions initially and after practice? Journal of Experimental Psychology: Learning, Memory, \& Cognition, 19, 922-930.

Proctor, R. W., \& Reeve, T. G. (1985). Compatibility effects in the assignment of symbolic stimuli to discrete finger response. Journal of Experimental Psychology: Human Perception \& Performance, 11, 623-639.

Proctor, R. W., \& Reeve, T. G. (1986). Salient-feature coding operations in spatial precuing tasks. Journal of Experimental Psychology: Human Perception \& Performance, 12, 277-285.

Proctor, R. W., \& Reeve, T. G. (Eds.) (1990). Stimulus-response compatibility: An integrated perspective. Amsterdam: North-Holland.

Reeve, T. G., Proctor, R. W., Weeks, D. J., \& Dornier, L. (1992). Salience of stimulus and response features in choice-reaction tasks. Perception \& Psychophysics, 52, 453-460.

Shaffer, L. H. (1965). Choice reaction with variable S-R mapping. Journal of Experimental Psychology, 70, 284-288.

VU, K.-P. L., \& Proctor, R. W. (2001). Determinants of right-left and top-bottom prevalence for two-dimensional spatial compatibility. Journal of Experimental Psychology: Human Perception \& Performance, 27, 813-828.

Vu, K.-P. L., Proctor, R. W., \& Pick, D. F. (2000). Horizontal versus vertical compatibility: Right-left prevalence with bimanual responses. Psychological Research/Psychologische Forschung, 64, 25-40. 


\begin{tabular}{|c|c|c|c|c|}
\hline $\begin{array}{r}\text { Mean Rea } \\
\text { and Mean Percen } \\
\text { Function of V } \\
\text { Compatibi } \\
\end{array}$ & $\begin{array}{l}\text { PEND } \\
\text { Times } \\
\text { of Er } \\
\text { l Com } \\
\text { nstruc }\end{array}$ & $\begin{array}{l}\text { A } \\
\text { Milli } \\
\text { in Ex } \\
\text { ibility } \\
\text { s, anc }\end{array}$ & $\begin{array}{l}\text { nds) } \\
\text { imen } \\
\text { rizor } \\
\text { ience }\end{array}$ & \\
\hline & & rizont & mpati & \\
\hline & & tible & Inc & $\overline{\text { tible }}$ \\
\hline Vertical Compatibility & RT & $\mathrm{PE}$ & RT & PE \\
\hline & l-Salien & splay & & \\
\hline $\begin{array}{l}\text { Vertical instructions } \\
\text { Compatible } \\
\text { Incompatible }\end{array}$ & $\begin{array}{l}280 \\
355\end{array}$ & $\begin{array}{l}0.94 \\
1.41\end{array}$ & $\begin{array}{l}320 \\
353\end{array}$ & $\begin{array}{l}1.48 \\
2.27\end{array}$ \\
\hline $\begin{array}{l}\text { Horizontal instructions } \\
\text { Compatible } \\
\text { Incompatible }\end{array}$ & $\begin{array}{l}302 \\
299\end{array}$ & $\begin{array}{l}0.72 \\
0.78\end{array}$ & $\begin{array}{l}329 \\
343\end{array}$ & $\begin{array}{l}2.19 \\
2.11\end{array}$ \\
\hline & ivalent I & & & \\
\hline $\begin{array}{l}\text { Vertical instructions } \\
\text { Compatible } \\
\text { Incompatible }\end{array}$ & $\begin{array}{l}290 \\
330\end{array}$ & $\begin{array}{l}0.70 \\
1.95\end{array}$ & $\begin{array}{l}319 \\
360\end{array}$ & $\begin{array}{l}1.02 \\
1.33\end{array}$ \\
\hline $\begin{array}{l}\text { Horizontal instructions } \\
\text { Compatible } \\
\text { Incompatible }\end{array}$ & $\begin{array}{l}296 \\
292\end{array}$ & $\begin{array}{l}0.39 \\
0.86\end{array}$ & $\begin{array}{l}339 \\
362\end{array}$ & $\begin{array}{l}2.34 \\
1.80\end{array}$ \\
\hline & tal-Sali & isplay & & \\
\hline $\begin{array}{l}\text { Vertical instructions } \\
\text { Compatible } \\
\text { Incompatible }\end{array}$ & $\begin{array}{l}292 \\
334\end{array}$ & $\begin{array}{l}0.55 \\
1.02\end{array}$ & $\begin{array}{l}349 \\
386\end{array}$ & $\begin{array}{l}2.27 \\
2.42\end{array}$ \\
\hline $\begin{array}{l}\text { Horizontal instructions } \\
\text { Compatible } \\
\text { Incompatible }\end{array}$ & $\begin{array}{l}304 \\
302\end{array}$ & $\begin{array}{l}0.78 \\
0.70\end{array}$ & $\begin{array}{l}362 \\
364\end{array}$ & $\begin{array}{l}1.64 \\
1.25\end{array}$ \\
\hline
\end{tabular}

APPENDIX B

Mean Reaction Times (in Milliseconds) and Mean

Percentages of Errors in Experiment 2 as a Function of Vertical Compatibility, Horizontal Compatibility, Instructions, and Display-Response Salience

\begin{tabular}{|c|c|c|c|c|}
\hline \multirow[b]{3}{*}{ Vertical Compatibility } & \multicolumn{4}{|c|}{ Horizontal Compatibility } \\
\hline & \multicolumn{2}{|c|}{ Compatible } & \multicolumn{2}{|c|}{ Incompatible } \\
\hline & RT & PE & RT & PE \\
\hline \multicolumn{5}{|c|}{ Vertical-Salient Display-Vertical-Salient Responses } \\
\hline \multicolumn{5}{|l|}{ Vertical instructions } \\
\hline Compatible & 340 & 1.48 & 322 & 1.09 \\
\hline Incompatible & 413 & 1.97 & 399 & 1.72 \\
\hline \multicolumn{5}{|l|}{ Horizontal instructions } \\
\hline Compatible & 319 & 1.41 & 349 & 2.11 \\
\hline Incompatible & 366 & 1.41 & 398 & 2.11 \\
\hline \multicolumn{5}{|c|}{ Vertical-Salient Display-Horizontal-Salient Responses } \\
\hline \multicolumn{5}{|l|}{ Vertical instructions } \\
\hline Compatible & 287 & 0.55 & 315 & 2.03 \\
\hline Incompatible & 375 & 2.50 & 362 & 1.64 \\
\hline \multicolumn{5}{|l|}{ Horizontal instructions } \\
\hline Compatible & 291 & 0.39 & 345 & 1.41 \\
\hline Incompatible & 302 & 0.86 & 363 & 1.64 \\
\hline \multicolumn{5}{|c|}{ Horizontal-Salient Display-Vertical-Salient Responses } \\
\hline \multicolumn{5}{|l|}{ Vertical instructions } \\
\hline Compatible & 334 & 1.33 & 338 & 0.70 \\
\hline Incompatible & 375 & 1.33 & 421 & 2.11 \\
\hline \multicolumn{5}{|l|}{ Horizontal instructions } \\
\hline Compat & 340 & 1.17 & 369 & 1.72 \\
\hline Incompatible & 354 & 2.19 & 408 & 3.36 \\
\hline
\end{tabular}


APPENDIX B (Continued)

\begin{tabular}{|c|c|c|c|c|}
\hline \multirow[b]{3}{*}{ Vertical Compatibility } & \multicolumn{4}{|c|}{ Horizontal Compatibility } \\
\hline & \multicolumn{2}{|c|}{ Compatible } & \multicolumn{2}{|c|}{ Incompatible } \\
\hline & RT & $\mathrm{PE}$ & RT & $\mathrm{PE}$ \\
\hline \multicolumn{5}{|c|}{ Horizontal-Salient Display-Horizontal-Salient Responses } \\
\hline \multicolumn{5}{|l|}{ Vertical instructions } \\
\hline Compatible & 314 & 0.31 & 368 & 1.17 \\
\hline Incompatible & 344 & 0.86 & 395 & 1.33 \\
\hline \multicolumn{5}{|l|}{ Horizontal instructions } \\
\hline Compatible & 275 & 0.94 & 331 & 2.42 \\
\hline Incompatible & 285 & 0.63 & 351 & 2.58 \\
\hline
\end{tabular}

\section{APPENDIX C}

Mean Reaction Times (in Milliseconds) and Mean

Percentages of Errors in Experiment 3 as a Function of Vertical Compatibility, Horizontal Compatibility, Instructions, and Display-Response Salience

\begin{tabular}{|c|c|c|c|c|}
\hline \multirow[b]{3}{*}{ Vertical Compatibility } & \multicolumn{4}{|c|}{ Horizontal Compatibility } \\
\hline & \multicolumn{2}{|c|}{ Compatible } & \multicolumn{2}{|c|}{ Incompatible } \\
\hline & RT & $\mathrm{PE}$ & RT & PE \\
\hline \multicolumn{5}{|c|}{ Vertical-Salient Display-Vertical-Salient Responses } \\
\hline \multicolumn{5}{|l|}{ Vertical instructions } \\
\hline Compatible & 357 & 0.70 & 380 & 1.64 \\
\hline Incompatible & 430 & 2.04 & 464 & 3.36 \\
\hline \multicolumn{5}{|l|}{ Horizontal instructions } \\
\hline Compatible & 356 & 0.70 & 381 & 2.58 \\
\hline Incompatible & 398 & 1.48 & 461 & 2.89 \\
\hline \multicolumn{5}{|c|}{ Vertical-Salient Display-Horizontal-Salient Responses } \\
\hline \multicolumn{5}{|l|}{ Vertical instructions } \\
\hline Compatible & 338 & 0.47 & 357 & 1.25 \\
\hline Incompatible & 391 & 1.88 & 429 & 2.89 \\
\hline \multicolumn{5}{|l|}{ Horizontal instructions } \\
\hline Compatible & 330 & 1.25 & 387 & 2.66 \\
\hline Incompatible & 343 & 1.33 & 415 & 2.66 \\
\hline \multicolumn{5}{|c|}{ Horizontal-Salient Display-Vertical-Salient Responses } \\
\hline \multicolumn{5}{|l|}{ Vertical instructions } \\
\hline Compatible & 349 & 0.94 & 382 & 1.88 \\
\hline Incompatible & 403 & 2.03 & 449 & 2.97 \\
\hline \multicolumn{5}{|l|}{ Horizontal instructions } \\
\hline Compatible & 341 & 0.78 & 382 & 2.34 \\
\hline Incompatible & 355 & 1.56 & 426 & 2.66 \\
\hline \multicolumn{5}{|c|}{ Horizontal-Salient Display-Horizontal-Salient Responses } \\
\hline \multicolumn{5}{|l|}{ Vertical instructions } \\
\hline Compatible & 347 & 0.78 & 395 & 1.33 \\
\hline Incompatible & 377 & 0.94 & 445 & 2.58 \\
\hline \multicolumn{5}{|l|}{ Horizontal instructions } \\
\hline Compatible & 325 & 0.63 & 400 & 2.34 \\
\hline Incompatible & 346 & 0.86 & 414 & 1.80 \\
\hline
\end{tabular}

(Manuscript received December 13, 2000; revision accepted for publication September 19, 2001.) 